\title{
UN PERFIL ADMINISTRATIVO PARA EL GERENTE PÚBLICO EN COSTA RICA
}

\author{
ANABELLE CASTILLO LÓPEZ \\ Escuela de Administración \\ Universidad Estatal a Distancia, Costa Rica \\ acastillo@uned.ac.cr
}

\section{RESUMEN}

Se encuentran muchos estudios, propuestas y especulaciones sobre las malas actuaciones del gobierno, con lo cual se han generado diferentes proposiciones para reformar el estado y/o el gobierno. Partiendo de ello, el presente trabajo aporta en esta problemáti$c a$, pero no desde la perspectiva de lo que debe ser la administración pública, ni de las grandes reformas al Estado sino desde lo que deben ser las competencias de un gerente público en Costa Rica. La premisa fundamental es que no se pueden efectuar cambios, grandes o pequeños, si quienes dirigen las instituciones no se encuentran preparados para realizarlos, por lo que se efectuó un análisis sobre las herramientas administrativas que el gerente público debe dominar para desempeñarse con altas posibilidades de éxito en la ejecución de los grandes proyectos nacionales. Eso llevó a determinar que el gerente público al igual que el del sector privado, necesita dominar el proceso administrativo pero adaptándolo a la dimensión que requiere el contexto de dicho sector, y a partir de aqui se derivaron las funciones, habilidades y roles que éste debe poseer y que no son diferentes a las del gerente en el sector privado excepto como ya se dijo, por las dimensiones y características propias que lo distingue. Para ello se realizó un análisis cualitativo sobre las posiciones de quienes sostienen que deben existir diferencias entre los enfoques administrativos para el sector público y el privado y para cada contexto en particular, sobre las propuestas realizadas para reformar el estado costarricensey sobre los enfoques que afirman que los resultados del gerente dependen del tipo de liderazgo.
PALABRAS CLAVE: GERENCIA PÚBLICA, PROCESO ADMINISTRATIVO, ROLES ADMINISTRATIVOS, LIDERAZGO, SECTOR PÚBLICO, SECTOR PRIVADO, REFORMA DEL ESTADO.

\section{ABSTRACT}

The main goal of this research is to offer an appropriate administrative profile for a high-level manager working in the Costa Rican public sector. There are many studies about administrative governance, about government results or approaches regarding government re-organization; however, it is difficult to find a research focusing on what is required for a manager to reach a high level of efficiency and efficacy in the public sector. The fundamental idea is that, in a country like Costa Rica, good governance cannot be achieved through the public sector (in the form of national programs), if public managers do not have administrative skills that allow them to do so. Therefore, this study focused on describing the skills needed by all public managers. It was necessary to evaluate, not only studies related to government organization in general, but also academic research related to this topic. In this sense, it was important to review those studies that pointed out that it is not possible to apply the same theoretical framework to public and private managers; those that pointed out that differences between environments do not allow the application of the same theoretical recipe; and those that gave more importance to leadership than to the main managerial skill to deliver positive results in the public sector. The qualitative research methodology of this study included the analysis of these previous studies and, at the same time, by discarding or accepting of proposals, the building of a profile for a public manager. It is shown, as 
a result, that the administrative process - planning, organizing, directing and controlling-offers the conditions under which a public manager must work. From this point of view, it is possible to conclude that theories can be applied to both public and private managers but that such theories must be adapted according to the specific characteristics of each context.

KEYWORDS: PUBLIC MANAGEMENT, ADMINISTRATIVE PROCESS, ADMINISTRATIVE ROLES, LEADERSHIP, PUBLIC SECTOR, PRIVATE SECTOR, GOVERNMENT REFORM.

\section{INTRODUCCIÓN}

Actualmente buena parte de la población, que acostumbre a leer la prensa, ha obtenido elementos para formarse un criterio acerca de la gestión gubernamental, puesto que, en este tipo de medios, se atribuyen una serie de ineficacias a la administración pública tales como la calidad de la educación, la calidad de la infraestructura vial, la inseguridad ciudadana, los problemas del medio ambiente, entre otros. Junto a ello, deben sumarse los permanentes calificativos, en cuanto a negocios o actuaciones del Estado, resultado de la actuación de los gerentes públicos. Basta abrir diariamente cualquier periódico o noticiero a nivel nacional para darse cuenta de cómo reiteradamente, se afirma, que la causa de esta problemática obedece a una administración pública que ya no ofrece respuestas a los problemas nacionales, por lo que es indispensable un cambio radical en las prácticas administrativas gubernamentales.

Para comenzar este análisis es necesario plantear que la problemática de la gestión pública es, a todas luces, multifactorial, y abordarla integralmente obligaría a estudiarla desde muy diversos ángulos. Por lo tanto, es a partir de reconocer esa integralidad que el presente trabajo trata de ofrecer aportes en un campo específico: en torno a la óptica de la gestión gerencial, donde se pretende determinar concretamente cuál es un perfil administrativo general para un gerente público. No se trata entonces de un estudio sobre la administración pública, sino del gerente público y -específicamente- de cómo éste realiza sus funciones y cuál es el comportamiento administrativo que debería seguir para organizar la institución a su cargo y brindar los servicios que, por disposición del gobierno de turno y dentro del marco legal vigente, le corresponde ejecutar.

La premisa fundamental de este trabajo, es que no se pueden llevar a cabo ni pequeñas ni grandes reformas si quien está a la cabeza de una institución, es decir, el gerente público, no entiende cómo ejecutarlas, cómo diseñar o coordinar el diseño de sistemas a nivel regional, sectorial o de la propia institución que dirige. Lo que debe importar a todo gerente es alcanzar un cierto nivel de eficacia con la mayor eficiencia posible. Para conseguirlo requiere echar mano de una serie de herramientas administrativas y conocimientos indispensables, que le permitan obtener los resultados esperados.

También es fundamental aclarar que, como se verá más adelante, el resultado del análisis que aquí se hace, no exige entrar en un debate sobre teoría de la administración, sino de tomar aquellos postulados más importantes con los que, un gerente público del más alto nivel, podría lograr los objetivos propuestos.

Tampoco puede sustraerse, un estudio como este del contexto que lo rodea; es decir, de la realidad costarricense, por lo que el resultado que se obtendrá será aplicable a este contexto, aunque eso no exime que pueda funcionar en otros.

Es así como, al delimitar cuáles son los ejes de trabajo de un gerente estratégico, nos referiremos a gerentes estratégicos del gobierno de Costa Rica; es decir, ministros, viceministros, presidentes ejecutivos de instituciones autónomas o empresas públicas costarricenses (con independencia del campo de acción). 
Asimismo, no se estudian las competencias de gerentes de nivel medio o bajo, en donde es probable que los requerimientos sean más técnicos.

\section{ANTECEDENTES}

Dentro de las respuestas, que los gobiernos costarricenses han ofrecido a las diversas dificultades de la administración pública, se encuentra la formación de comisiones para el estudio de los problemas que ésta plantea. Basta mirar los trabajos de reforma administrativa y reforma del Estado, planteados desde la década de los ochentas, para darse cuenta de que existe una necesidad por mejorar muchos aspectos. Las respuestas dadas van con dirección a ofrecer una reforma profunda en la administración pública y/ o en el Estado costarricense.

Uno de los esfuerzos más importantes, ha sido el realizado por la Comisión Consultiva para la Reforma del Estado conocida como COREC I. Dicha comisión fue una iniciativa del Ministro de Planificación Nacional y Política Económica, quien, en 1989, integró la misma con expertos administradores, juristas, economistas, científicos sociales y profesionales de otras disciplinas; y su gestión culminó en 1990 con la elaboración de estudios y diagnósticos. (COREC (1990).

Los fines principales, de la COREC I, fueron realizar estudios tendientes a:

a) Redistribuir el poder político entre los estamentos de la sociedad, a fin de profundizar el régimen de la democracia.

b) Reestructurar la organización del Estado para darle mayor flexibilidad, eficiencia y productividad en el logro de sus objetivos (MIDEPLAN, 2012).

Posteriormente, en el período del 2007 al 2010, se formó la CEARE (Comisión de Eficiencia Administrativa y Reforma del Estado), la cual estudió el tema de la eficiencia administrativa y de la reforma del Estado. Esta comisión -integrada por políticos, académicos y funcionarios públicosse subdividió en tres grupos de trabajo para desarrollar temas de indiscutible relevancia para el país; además, consideró que no se abocarían a un trabajo de reforma integral del Estado, tal y como se había trabajado en estudios anteriores; sino que su planteamiento sería hacia lo micro, tendiente a mejorar la prestación de servicios a los ciudadanos. Con tal idea, se desarrollaron los siguientes temas:

\section{Empleo público}

\section{Planificación, presupuesto y control}

\section{Organización de la Administración Pública}

Además de estos tres temas, la Comisión se propuso realizar aportes en algunas áreas específicas; con el fin de que el país pudiera enfrentar algunos desafíos apremiantes como:

- Superar la visión cortoplacista, desarrollando un proceso de planificación estratégica, para lo cual propone fortalecer a MIDEPLAN como institución rectora en este campo.

- Desarrollar un sistema de información para la toma de decisiones en lo referente al diseño y ejecución de las políticas públicas.

- Mejorar la calidad de los servicios.

- Elevar las capacidades de los funcionarios públicos para la prestación de los servicios, dentro de lo cual se propone crear condiciones para que los funcionarios conozcan la normativa, desarrollen un sistema de evaluación de desempeño (que motive a los buenos funcionarios), que establezcan incentivos para los buenos funcionarios, que desarrollen estándares de calidad para el ingreso y permanencia de los funcionarios. 
- Optimizar los procesos de contratación administrativa.

En general, estas recomendaciones tienden al ordenamiento jurídico y administrativo sin ahondar en el cómo llevarlo a cabo. Tal y como lo indica MIDEPLAN, muchas de las soluciones se plantean como decretos, para obligar a las instituciones a desarrollar sus funciones de manera diferente, a desarrollar sistemas de información, capacitación a funcionarios, entre otros (MIDEPLAN, 20012).

Finalmente, se crea la Comisión de Eficiencia Administrativa (CEA), que fue integrada mediante el Decreto Ejecutivo No36175-PLAN del 17 de agosto de 2010, presidida por el Ministerio de Planificación Nacional y Política Económica (MIDEPLAN) y que operó como un espacio de discusión, análisis y producción de insumos; con el propósito de asesorar al ministro para mejorar la eficiencia en la Administración Pública costarricense. El decreto que creó esta comisión tiene la base legal en una instancia de coordinación y asesoría, que había sido creada en el artículo 17 de la Ley de Planificación Nacional No5525 de 2 de mayo de 1974.

Como resultado de esto, se encuentra una serie de documentos de análisis, en temas como los siguientes:

- Promoción de una cultura de planificación estratégica para el desarrollo a largo plazo.

- Contribución con el mejoramiento e integración de los sistemas nacionales de planificación, presupuesto, información, evaluación e inversión pública.

- Recomendaciones de acciones para fortalecer las potestades de tutela administrativa o dirección intersubjetiva.

- La generación de procedimientos para la simplificación de las estructuras y coordinación intra e interinstitucional.
- Recomendación de acciones que permitan agilizar la ejecución de obras públicas.

- Sugerencias de alternativas que permitan optimizar el uso de los recursos financieros disponibles.

- Proposición de mecanismos que permitan mejorar las habilidades y destrezas del recurso humano. (MIDEPLAN, 2011)

Cada uno de los grupos presentó sus conclusiones; de acuerdo con su marco conceptual de investigación y aplicaron diferentes criterios, para valorar lo que-consideraron- debían ser los cambios en la gestión pública. A pesar de todos estos valiosos aportes, no se han visto grandes mejoras en la prestación de servicios en la administración pública. La mayor parte de los cambios dados fueron el resultado de los planteamientos de la COREC de los años ochentas, relacionados con la concepción de Estado, y se orientaron a la eliminación y apertura de actividades que tradicionalmente eran monopolio estatal.

Es probable que la dificultad, que presentan todos esos valiosos aportes, haya estado en lo complejo de su ejecución. En primer lugar, la visión integral con que se plantea la problemática los convierte de por sí en un discurso difícil de llevar a la práctica. En segundo lugar, la ausencia de procedimientos y cronogramas de ejecución que comprometan, a los gobiernos proponentes y a los subsiguientes, a llevar a cabo las transformaciones, también han sido factores que no han ayudado a ejecutar algunas de esas reformas.

Arribamos entonces al tema que ocupa este estudio y es la gerencia pública que podía hacer posible el logro de dichas propuestas, superando esas barreras administrativas. Es decir, debido a la complejidad propia de los planteamientos integrales, se requería de una gerencia pública que le diera agilidad y que pudiera liderar este tipo de cambios. 
No se puede modernizar el aparato público si no existen gerentes que ejecuten la propuesta, que puedan modernizar cada institución, ajustándola a las necesidades. Así, por ejemplo, no se puede lograr un proceso de planificación en cada institución como lo solicitan las propuestas, si los únicos que conocen cómo hacerlo son los especialistas del Ministerio de Planificación, porque lo que se requiere es que sea la propia institución que, conociendo su organización y su sector, diseñe y lleve a cabo sus propios planes. Es así como este proceso de planificación en cada institución requiere entonces de un gerente que lo dirija, lo organice y lo controle.

Una de las grandes ausencias en todos estos planeamientos es el abordaje del tema de la gerencia pública. El tratamiento de éste se realiza en general desde el punto de vista de la gerencia a nivel nacional, pero no se visualiza la problemática desde el punto de vista del gerente en cada institución. Ello a pesar de que es indiscutible que, para alcanzar los objetivos esperados, se requiere de una ejecución expedita que dependerá de las buenas prácticas del gerente. Curiosamente, cuando se trata el tema de gerencia, las propuestas giran alrededor de los sistemas de planificación, presupuesto y evaluación nacional, y sólo en alguno de los puntos, sobre todo en temas relacionados con obra pública, se hace alusión a los gerentes de proyecto. Lo que sí queda claro, dentro de esas propuestas, es la manifiesta necesidad de una buena gestión dentro de las instituciones.

Aunado a lo anterior, la Ley de Planificación Nacional (número 5525 del 2 de mayo de 1974, en su artículo número 16) dispone que los ministerios e instituciones autónomas y semiautónomas lleven a cabo una labor sistemática de modernización de su organización y procedimientos, en procura de aumentar la eficiencia y productividad de sus actividades, y cumplir así, con los objetivos que persigue el Sistema Nacional de Planificación. No obstante, de nuevo, aún la obligatoriedad de la ley no ha dado un verdadero cambio, y no podemos afirmar que exista un efectivo desarrollo de una cultura de planificación.

Meoño (2012, p. 10), refiriéndose a la Ley de Planificación de 1974 en Costa Rica dice que esa ley tiene todo: calidad teórico-conceptual e instrumental, pero no tiene la fórmula "mágica" que hiciera que nuestros líderes, la entendieran.

Efectivamente, ello es así, entre otras cosas porque los temas de la gerencia pública no son comúnmente tratados en los planes de las reformas costarricenses, aún cuando el contexto gubernamental se ha encontrado inmerso en una gran confusión, producto de las demandas constantes y urgentes de la ciudadanía por satisfacer sus necesidades, lo que exige técnicas de gestión modernas.

Como puede observarse, existen las más diversas opiniones sobre cómo mejorar la acción del gobierno siendo finalmente los medios de comunicación los que, al menos en nuestro contexto, se han convertido, en la mayor influencia de los ciudadanos para juzgar la realidad nacional.

El reto es entonces ofrecer un aporte a esta temática partiendo de la premisa de que el gerenciamiento es fundamental para el éxito de los organismos públicos. Es decir, la administración pública se desenvuelve en un entorno que tiene una serie de desafíos políticos, procesos jurídicos complejos y culturas diversas y para enfrentarlos se requiere de una gran capacidad gerencial.

\section{METODOLOGÍA}

Esta es una investigación cualitativa, que construye un perfil administrativo general para el gerente público costarricense a partir de un recorrido por las principales propuestas administrativas o teórico-administrativas realizadas para reformar el estado y el gobierno costarricense desde los años ochenata, así como de algunas otras referencias teóricas, nacionales e interna- 
cionales. El objetivo es buscar en ellas la importancia que debe tener el gerente como ejecutor de propuestas políticas, y las herramientas administrativas que le permitan conseguir ciertos resultados. La metodología consiste en analizar tales propuestas y paralelamente ir comprobando si se requiere un perfil administrativo general para esos funcionarios, si existe alguna diferencia entre la gerencia del sector público y la del sector privado y determinar si dentro de la teoría existente se puede obtener el perfil,-funciones y roles administrativos- que debe desempeñar un gerente en el sector público costarricense con el fin de ejecutar con razonable eficiencia y eficacia los grandes proyectos nacionales.

\section{EL PERFIL DEL GERENTE}

El perfil gerencial público que se requiere, es aquel indispensable para construir un gobierno, orientado a ofrecer respuestas a las demandas de la ciudadanía, por lo que el gerente debe interpretar esas demandas, visualizar el futuro a partir de ese contexto y dirigir las distintas instituciones hacia la consecución de esos bienes y servicios.

El gerente entonces debe ser capaz de agenciar los recursos y organizar las instituciones para la obtención de los bienes y servicios. El tipo de organización puede ser más o menos participativa, más o menos abierta, más o menos descentralizada; todo depende del contexto en que se desenvuelve y de los recursos de que dispone, no existen recetas sobre el tipo de organización en cada contexto, pero lo importante es obtener en cualquiera de ellas un nivel aceptable de eficacia con un nivel adecuado de eficiencia.

Lo que aquí se plantea es una visión del gerente, para generar el cambio en la Administración Pública. El gerente que se requiere desde esta perspectiva, es aquel que basa su poder e influencia en los resultados de su gestión en la sociedad a la cual sirve. No se trata del tipo de gerentes que logra resultados orientados a favo- recer el grupo que lo impulsó a la cúspide, sino aquel que cumple con las demandas de la población que paga su salario.

La idea fundamental es que los cambios profundos se darán en la medida en que se den los cambios en los individuos, y dentro de las organizaciones se debe comenzar por las más altas autoridades, porque éstas serán el ejemplo y el motor que impulsa el cambio para la totalidad de la institución que dirige.

Si bien es sólo desde este cambio individual que se pueden obtener resultados, también es cierto que no es posible lograrlo sólo con cambios de actitud, sino que se requiere de un conjunto de conocimientos que hagan posible los cambios que se necesitan para cada situación. No se trata tampoco de un proceso de profesionalización, puesto que no toda profesionalización puede ser útil para generar un cambio en los niveles gerenciales, sino que debemos buscar las competencias idóneas para lograr tales cambios.

Es a partir del análisis anterior que nace la necesidad de preguntarse ¿cuál es el perfil de un gerente público?, Qué conocimientos debe tener el gerente público? Qué diferencia existe entre éste y el gerente del sector privado? Cuáles son las funciones del gerente público?

Para dirigir cualquier institución u organización, tanto pública como privada, se necesita, efectivamente, conocimiento en el propio campo de acción de la institución pero eso no es suficiente. Como ya fue dicho, es indispensable además contar con conocimientos sobre los principios gerenciales que deben ser aplicados según el contexto en que se desenvuelve cada organización. Es decir, el gerente necesita disponer de herramientas administrativas que le permitan resolver asuntos cotidianos y no tan cotidianos relacionadas con el diseño de organizaciones, el diseño de operaciones y procedimientos y la guía, motivación y control de las funciones del sector a cargo (Daft, 2011). 
Visto así, la carencia de herramientas administrativas básicas para dirigir una institución conduce a deficiencias en la ejecución de la visión del país, puesto que detiene el avance de los programas nacionales de desarrollo.

Por otra parte, debe tomarse en consideración que, si bien la gerencia requiere de un conjunto de instrumentos administrativos, formados por teorías o enfoques y sustentados en ciertos principios y justificaciones; tampoco se puede afirmar que el conocimiento de ellos, por sí mismos, sea garantía de la buena ejecución pero, lo que sí es posible afirmar, es que sin su utilización no se puede lograr eficiencia y eficacia en la gestión.

Por lo tanto, tratándose de la gestión pública, ésta, al igual que la privada, debe lograr un mayor compromiso con la aplicación de técnicas para mejorar la racionalidad, para conseguir un mejor aprovechamiento de los recursos materiales, financieros y humanos y, por tanto, alcanzar superiores resultados.

Para construir este marco teórico general que se busca, en este trabajo se procede a reconocer qué se espera de un gerente público de alto nivel para luego determinar dentro de los enfoques de administración, aquellos relacionados con las funciones del gerente. Una vez definidos esos enfoques, se valorará si son aplicables al sector público de manera que se puedan alcanzar con esas herramientas, grados aceptables de eficiencia y eficacia, porque, tal y como lo afirma Bolaños, no se trata de que el gobierno funcione como una empresa, sino de que adopte un talante más ejecutivo (Bolaños 2008, p 144).

Por lo tanto, siguiendo con ese análisis el primer paso es reconocer qué se espera de un gerente público de alto nivel. En este sentido se tienen tres grandes aspectos a considerar:

- diferencia entre lo público y lo privado

- el conjunto de conocimientos necesarios y las funciones que debe desempeñar

\section{Diferencias entre lo público y lo privado}

En este apartado se hará una descripción de las principales diferencias entre el sector público y el privado que deben ser reconocidas por todo gerente, según el contexto en que se desenvuelve.

La primera gran diferencia, entre ambos sectores, es que, mientras el sector público tiene fines sociales que cumplir, y la mayoría de sus costos son pagados por impuestos o contribuciones de los ciudadanos; el privado, tiene objetivos de lucro sin los cuales no logra sobrevivir. Por lo tanto, mientras las acciones del primer sector se dirigen hacia el cumplimiento de objetivos, lo cuales a su vez deben estar orientados a satisfacer las necesidades de los ciudadanos, las acciones del segundo se dirigen a la obtención de beneficios económicos, por medio de la venta de bienes y servicios a los ciudadanos. Es necesario aclarar en este punto que aunque también existen negocios públicos, creados por razones estratégicas para el estado, sin embargo, la primera gran diferencia entre ambos sectores se encuentra en los objetivos de lucro de éste último.

La segunda gran diferencia entre el sector público y el privado es que mientras el Estado y sus administraciones constituyen un sistema integrado, no ocurre así en el sistema disperso de organizaciones privadas o empresariales (Meoño, 2010).

Esta idea además la instituye el artículo 50 de la Constitución Política de Costa Rica, cuando establece que el Estado procurará el bienestar de todos los ciudadanos, por lo que el ámbito de la Administración Pública, debe ser un sistema integrado que abarque la totalidad de los derechos de los habitantes a lo largo y ancho de todo el territorio nacional; mientras que la empresa privada, por grande y poderosa que sea, no produce el tipo de desarrollo asociado a todos los habitantes de un país, que sí debe tener 
la administración pública. Ello se aplica aun para las transnacionales que prestan un conjunto de bienes y servicios distribuidos en muchos territorios pero que no abarcan a todos los ciudadanos (Meoño, 2010).

En esas circunstancias la forma de planificar, dirigir, organizar y controlar, las instituciones en la administración pública, tiene dimensiones distintas a las de la empresa privada y, consecuentemente, para el diseño de los sistemas en las primeras, se requiere planteamientos que tomen en cuenta esa complejidad. El gerente público debe estar preparado para administrar en este contexto.

La tercera diferencia se refiere a que al estar la administración pública obligada a cumplir con un propósito social, el resultado, tanto de la eficacia como de la eficiencia, debe verse bajo un marco social.

Es decir, el Estado y su institucionalidad deben operar bajo elevados cometidos de eficacia o logro suficiente para cubrir las necesidades o demandas a que tienen derecho todos los habitantes, y lo que marca la diferencia con el sector privado, es que la eficiencia debe estar supeditada al logro de esa eficacia. Es así como en muchas ocasiones la eficiencia, o costos con que se deben asumir tales cometidos, contravienen las pautas o criterios utilizados por la empresa privada, ya que en la administración pública se hace necesario llevar a cabo tales acciones, aunque sea a un costo más alto. Los costos o riesgos que la empresa privada no asumiría porque incurriría en pérdidas, la empresa pública sí debe asumirlos, puesto que es indispensable para la existencia de la colectividad, aunque al lograr el nivel de eficacia demandado por la colectividad, la actuación del gerente público siempre debe tener presente la escogencia de los caminos menos onerosos.

En este sentido debe tener muy claro que el programa de partido, se debe convertir en el programa de gobierno, y que lo allí ofrecido, constituye la promesa que el partido ha realizado a los ciudadanos.

La cuarta diferencia, la indica el Meoño (2010) cuando describe la existencia de una diferencia entre el control ciudadano de la gestión pública y la libertad con que se ejerce la gestión privada:

Si bien la empresa privada enfrenta, y a veces acaba asfixiada, por el exceso de regulaciones, sobre todo aquellas anteriores a la creación de una empresa o actividad lucrativa, lo normal es que su devenir comercial o económico responda a una libertad casi ilimitada para producir, comprar y vender; mientras que en materia de Estado, cada institución, funcionario y jerarca, están sometidos al imperio de la Ley. De esta manera hay muchas más prohibiciones sobre el funcionario público que sobre el empleado o empresario privado. Es por esta razón que la rendición de cuentas en el contexto público es una obligación del funcionario y un derecho del ciudadano constitucionalmente normado (Meoño, 2010).

El gerente público, por lo tanto, debe estar plenamente consciente de estas diferencias, para no verse sorprendido por las implicaciones legales de sus actuaciones.

Finalmente a todo este planteamiento debe agregarse una última diferencia en el sentido de que el gerente público necesita conocer el contexto político en que se desenvuelven las instituciones públicas, porque esto afecta sobre todo el proceso de toma de decisiones y la dirección pública. Si bien la política es una actividad presente en cualquiera de las actividades, tanto del sector privado como del público, el medio en que se desenvuelve el gerente público es mucho más complejo, puesto que está expuesto a la dinámica política de todos los sectores de la sociedad. Se está, entonces, ante un conjunto de factores que no tienen parangón en el contexto privado. 
Adicionalmente, a todo lo hasta aquí descrito sobre las particularidades del contexto público que debe ser reconocido por el gerente, es indispensable, también, disponer de una serie de conocimientos teóricos, para poder desarrollar y ejecutar programas dentro de ese contexto de gran complejidad, y así liderar el área que se le encomienda dentro del sector público.

\section{Conocimientos administrativos y funciones básicas del gerente público}

Se trata, entonces, del segundo aspecto importante para definir el perfil del gerente público: el conjunto de conocimientos administrativos que debe tener éste para desempeñar su función.

De la misma manera que es fundamental para el éxito, entender y conocer el sector en el que se desenvuelve, y las diferencias entre lo público y lo privado, el gerente público debe conocer, además, sobre herramientas administrativas - que pueda aplicar en un contexto confuso- y estar así preparado para dominar la complejidad de los procesos de análisis, la toma de decisiones y la acción pública, que permitirían participar e influir más racional y agresivamente en las instituciones como tales.

Para seleccionar dentro de todo el marco teórico, cuáles son esas herramientas fundamentales, lo primero que se debe tener claro es: qué es un gerente y qué es específicamente un gerente público.

\section{Funciones generales de un gerente}

Es fundamental recordar que la administración es una de las actividades de más importancia de la sociedad y que la gerencia, o la cúspide de la organización, es la responsable directa de su funcionamiento.

Visto así: ¿Cuáles son las funciones básicas de un gerente?
Se tiene claro que el trabajo de los gerentes no es la ejecución de los procedimientos específicos empleados para llevar a cabo las acciones concretas, sino que se trata de la coordinación de todas las acciones que se desarrollan en la organización. Consecuentemente, debe definir cuál es el proceso seguido para organizar todo el trabajo, así como también las redes de coordinación que son necesarias desarrollar una estrategia y empujar su ejecución.

En este sentido, Drucker (citado por Robbins y Coukter, 2011) definió claramente lo que debe saber y lo que debe hacer la cúspide de la organización y dice que ellos deben estar involucrados primordialmente en el plan estratégico, en el desarrollo de la visión y/o misión de la empresa (u organización) y debe agregarse el hecho de que la cúspide de la organización estará siempre comprometida con los recursos financieros.

Para Drucker, lo que deben hacer los gerentes marca una diferencia, ya que éstos representan el eslabón indispensable para ligar la estrategia con la táctica, a la táctica con los procesos y procedimientos y, finalmente, estos últimos con las acciones de los participantes organizacionales.

\section{Conocimientos y funciones generales de un gerente público}

A partir de esta descripción de los deberes y saberes del gerente, se puede entonces indagar sobre lo que trata la gerencia pública.

Duque (2005) señala que:

El gerente público es un profesional altamente calificado que es empleado por el estado y está íntimamente involucrado en lo que ha dado en llamarse la profesión gubernamental...En otras palabras, es capaz de alcanzar altos niveles de eficiencia y productividad por parte del funcionamiento del Estado (p.1). 
Interesa, entonces, saber cuáles conocimientos administrativos debe tener el gerente para desempeñar esta profesión, cuáles son las funciones que le corresponde lleva a cabo para lograr esa eficiencia y eficacia.

Una respuesta a esa pregunta se encuentra en los escritos del Ex presidente José Figueres Ferrer, en el documento Cartas a un Ciudadano, donde nos brinda una definición de la gerencia pública a partir de toda su experiencia como mandatario, que no fue poca, y donde deja manifiesta la preocupación e importancia que él profesaba sobre este tema.

Estimado ciudadano:

Una de las cosas interesantes que voy aprendiendo en esta posición presidencial en que usted me ha colocado, es que el Gobierno cumple dos funciones distintas, que solemos confundir generalmente, cual si fueran una sola.

Estas dos funciones pueden llamarse, para distinguirlas con nombres diferentes, "administrar" "y "gobernar".

Administrar es dirigir el funcionamiento diario de los organismos públicos, tal cual se maneja una empresa particular o un negocio de tamaño grande. Repartir el trabajo organizadamente, ver que cada funcionario cumpla con su deber, percibir las entradas de dinero y hacer los gastos llevando buenas cuentas.

Gobernar es, en esta distinción que estoy haciendo, encauzar el esfuerzo nacional hacia la formación de una patria mejor, constituida por ciudadanos mejores, y que proporcione a sus hijos un ámbito de vida mejor. Gobernar democráticamente, ejerciendo el Poder por delegación, es orientar el país hacia el género de bienestar, de cultura y de moral a que sus ciudadanos aspiran.
...Ninguna de las dos responsabilidades es más importante que la otra. Ambas se complementan en el curso de la vida nacional, como en el viaje de la nave que complementa la misión del navegante, que determina los rumbos sobre los mapas, mirando a las estrellas, con la pericia del piloto que mantiene día y noche la dirección, por entre oleajes y vendavales (Figueres,1956).

Como puede observarse, quién mejor que el señor Expresidente, para entender con asombrosa claridad las funciones de lo que en este trabajo llamamos gerente público.

\section{Enfoques administrativos para el gerente en el sector público}

Diferencia o semejanza con el sector privado

Aunque a partir de la experiencia descrita en la presidencia se puede llegar a la conclusión de que las herramientas administrativas que el gerente público necesita son iguales a las del gerente en el sector privado, puesto que tal y como lo afirma el Expresidente Figueres, las funciones relacionadas con lo que él llama "administrar" son iguales a las del sector privado, es necesario hacer notar que, algunos estudiosos de la administración parecen no participar al menos en parte con esta idea.

Para ciertos autores, los enfoques elaborados para el sector privado, deben ser sometidos a investigación antes de ser aplicados al sector público, de la misma manera que enfoques elaborados en unos contextos no deben ser aplicados en otros. Es así como autores como Meoño (2012) y Cabrero (2004), desde sus respectivos trabajos, aseveran que no se deben copiar enfoques construidos en contextos diferentes para ser aplicados en el sector público.

Sin embargo aunque una primera lectura parece no dejar lugar a dudas de que se trata de po- 
siciones antagónicas, una lectura más detallada de esas afirmaciones muestran que en buena parte podrían no existir tales diferencias.

Existen dos aspectos importantes que debemos resaltar en la exposición de los autores mencionados. Por un lado, estos enfoques no dejan claro cuáles serían las diferencias en las funciones que se ejecuta en uno y otro sector que demuestren que las herramientas administrativas que requieren unos y otros sean diferentes; por otra parte, estos autores muestran coincidencia con lo expresado por el Expresidente en el sentido de que para ellos, la toma de decisiones en la gerencia pública, debe apoyarse en un proceso de trabajo lo más científico y metódico posible, respecto a lo que se necesita y lo que en realidad se dispone, tal y como lo trataba de hacer ver el señor Figueres.

Es decir que, es probable que ambas posiciones concuerden en que tanto la organización pública como la privada, tienen facetas como: una faceta mecanicista en sus procesos, una faceta de informalidad, de relaciones humanas, de sistemas, de calidad, entre otros.

Para todas estas facetas se han desarrollado enfoques teóricos generados en su mayoría a partir del estudio del contexto del sector privado y es probable que, en todos los enfoques de la teoría de administración que van surgiendo, podamos encontrar aplicabilidad en cada uno de esos sectores.

Por lo tanto, puede concluirse que tal y como lo afirmaba el Expresidente, los enfoques de administración que rigen la gerencia son los mismos, tanto para el sector público como para el privado y para cualquier contexto en el que el administrador se desenvuelve.

Desde esta perspectiva, entonces, no se debería ir por el camino de elaborar enfoques administrativos para cada país o región, puesto que los postulados básicos son generales ya que se basan en comportamientos humanos universales y pueden ser útiles en cualquiera de ellos, aunque hayan sido creados en contextos diferentes.

Para apoyar esta afirmación, se presenta en la Tabla 1 los enfoques en las ciencias administrativas, planteado por Cajas (2006), y al cual se ha agregado una pequeña síntesis explicativa de cada uno, donde se puede dar cuenta de que ningún enfoque administrativo, originado para el sector privado, es descartable para la administración pública.

TABLA1

\section{EVOLUCIÓN DEL ESTUDIO DE LA ADMINISTRACIÓN PÚBLICA}

\begin{tabular}{|l|l|c|}
\hline \multicolumn{1}{|c|}{ Enfoques } & \multicolumn{1}{|c|}{ Naturaleza } & $\begin{array}{c}\text { Período dominante } \\
\text { Aproximado }\end{array}$ \\
\hline Política-Administración & Apolítica & $1900-1925$ \\
\hline Ciencias Administrativas & Fundamento científico: planificación, dirección y selección científica. & $1920-1940$ \\
\hline Weberiano-burocrático & Predominio de las normas: organización, manuales, jerarquía & $1930-1970$ \\
\hline Humanista & Orientación humanista: equipos, liderazgo, motivación, remuneración. & $1960-1980$ \\
\hline Políticas Públicas & Orientación a las políticas públicas & $1970-1980$ \\
\hline Sistémico & Orientación al entorno & $1980-2000$ \\
\hline Nueva Gestión Pública & Orientación empresarial: reinvensión, outsoursing & $1980-2000$ \\
\hline
\end{tabular}

Fuente: adaptado de Cajas (2006, p.13) 
Al observar este recorrido por el desarrollo de las ciencias de la administración, nos lleva a sintetizar ambas posiciones, en el sentido de que, efectivamente, aunque existen diferencias entre lo público y lo privado, y entre los diferentes contextos, los enfoques propuestos no deben asumirse como dogmas y no pueden aplicarse de la misma manera en todo contexto ni en todo sector; si bien el enfoque es el mismo, la aplicación es diferente y cada contexto requerirá hacer los cambios necesarios.

No se debe olvidar que la administración es ciencia y es arte; y por lo tanto el arte de administrar requiere del ingenio para aplicar enfoques, o bien, para adaptarlos o redefinirlos según las limitaciones o las oportunidades del contexto. Por ejemplo, enfoques como descentralización y desconcentración pueden ser útiles y aplicarse de manera generalizada en países de amplios territorios, pero podrían generar dificultades en la toma de decisionesen países pequeños- si no se tiene el cuidado de desconcentrar o descentralizar sólo ciertos asuntos públicos, que requieren ser tramitados o decididos en las comunidades, y en su lugar, utilizar la moderna tecnología para tramitar de manera expedita otros asuntos públicos, que demandan una visión nacional integral y, consecuentemente, de manera más centralizada. Es decir, algunos asuntos pueden ejecutarse por las oficinas centrales, directamente con los usuarios, en cualquier región del país, utilizando así las ventajas y el ahorro de recursos que ofrece la tecnología.

Visto desde esta segunda óptica, se podría decir que la ejecución de los programas y proyectos, tanto en el sector público como en el privado, requieren de una suerte de investigación, acción para aplicar los enfoques teóricos a cada situación o contexto, por lo que se realiza en el mismo momento de la ejecución; es decir, requiere de una forma de cuestionamiento auto reflexivo, llevada a cabo por los propios participantes, con la finalidad de mejorar la racionalidad de la propia práctica, de mejorar el conocimiento de dicha práctica y de mejorar el contexto general en el que la acción se lleva a cabo.

En la figura 1, se presenta la espiral del investigador Kurt Lewin (INFED ,1946), quien señala los pasos que deben darse para llevar a cabo una investigación- acción a partir de una idea o marco teórico general. Indica, este autor, que el enfoque de investigación debe estar orientado a la solución de problemas, sobre todo en las áreas sociales o de ingeniería social, y que debe ser una especie de aprendizaje a partir de la experiencia. Es un círculo de aprendizaje en donde se parte, como ya fue dicho, de una idea general o de unos postulados teóricos generales en los cuales se identifican una serie de hechos importantes. A partir de ellos, se planea la forma de ejecución, y una vez que se lleva a cabo el primer paso, se evalúa los resultados para determinar las mejoras que se requieren, con el fin de corregir los errores y volver a tomar el segundo paso. De esta manera, por ejemplo, la ejecución de un programa en el sector público se debe convertir en un círculo de mejora continua, de prácticas que son corregidas, en lugar de aplicarlas tal y como se determinó inicialmente.

Esta forma de actuación es indispensable sobre todo en el sector público, en donde, como se ha dicho anteriormente, el gerente debe desafiar contextos de gran complejidad, lo que presenta dificultades aun mayores para prever, por medio de la elaboración de planes, la totalidad de los acontecimientos a enfrentar. 


\section{FIGURA 1 \\ ESPIRAL DEL INVESTIGADOR DE KURT LEWIS}
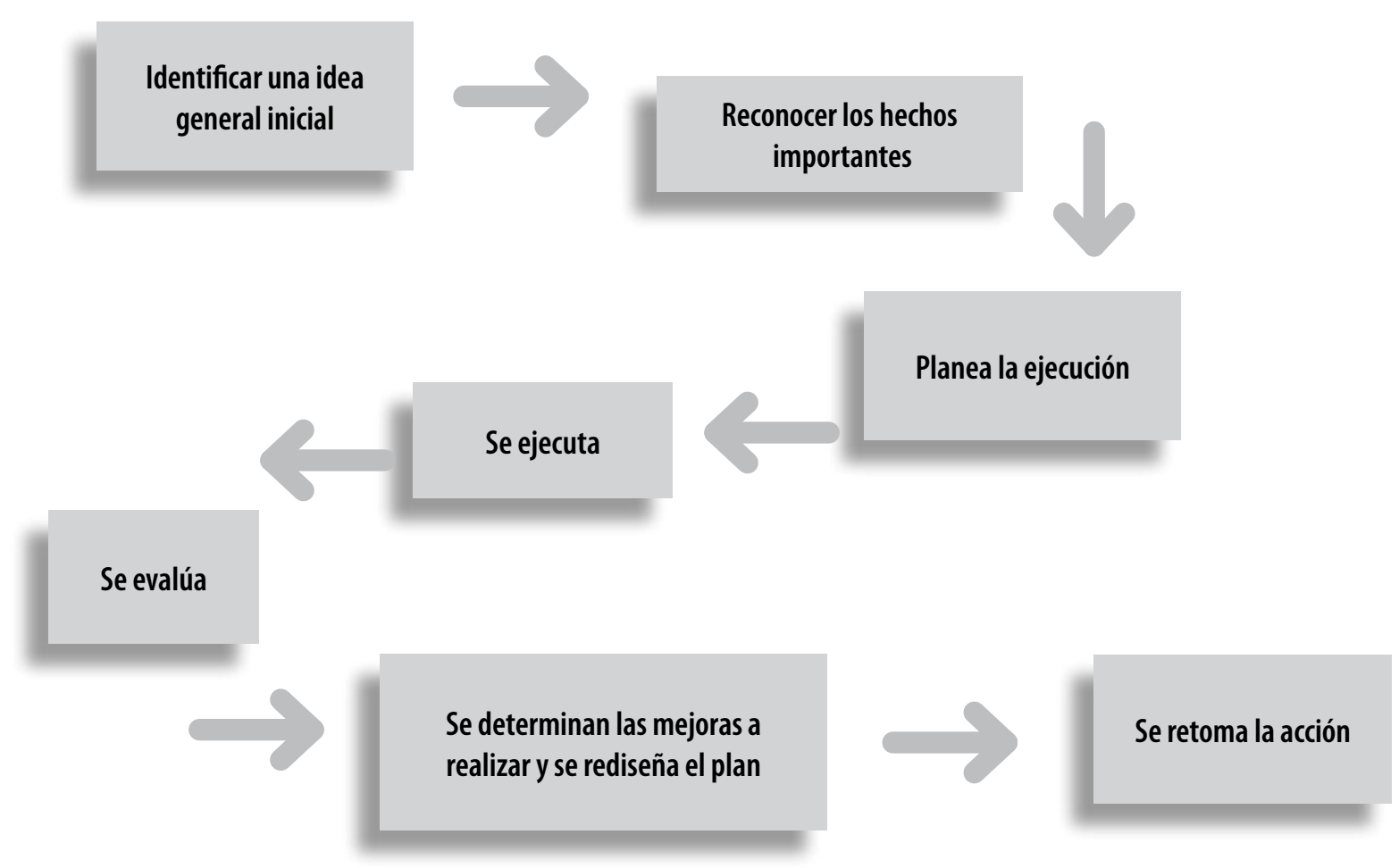

Fuente: tomado de the encyclopedia of informal education [www.infed.org].

\section{Enfoques administrativos del gerente público}

En consecuencia, el administrador público debe tener el conocimiento suficiente de distintos enfoques, para poder aplicarlos con la creatividad suficiente, de manera que pueda dar soluciones a los problemas en los diferentes contextos.

Lo anterior viene a reforzar la tesis aquí planteada de que en la administración, tanto pública como privada, se aplican los mismos enfoques administrativos, pero que en ningún caso caben modelos aplicables a toda situación y, por el contrario, el gerente público o privado debe aplicar su ingenio y construir soluciones, utilizando el marco teórico de que dispone -o aún creando uno nuevo a partir de éste-, pero siempre con la mira puesta en obtener la mayor eficacia y eficiencia posible dentro del contexto dado. Claro está que en el sector público, el marco legal delimita la libertad de acción, pero todavía dentro de esa restricción, el gerente debe buscar el camino más expedito para llevar a cabo las acciones, evaluando y reevaluando los resultados de ellas, reinventando como se diría en el enfoque de la Nueva Gestión Pública.( Michael Barzelay, 2000).

\section{Liderazgo versus gerencia}

Por otra parte, además de la anterior divergencia -en cuanto a si las herramientas administrativas deben ser diferentes en distintos sectores y contextos-, existen también variedad de criterios respecto a las funciones del gerente. En algunas investigaciones pareciera que lo importante no 
es el estilo administrativo, que pueda aplicar el gerente público, sino que, de manera contraria, la función del administrador debe estar orientada a construir un estilo de liderazgo que le permita mantener relaciones (al menos cordiales) con grupos de poder, con miembros de su propia institución y con los jerarcas superiores.

En este sentido se realizan los trabajos de Ayoub (2010), investigador que estudia la relación entre el estilo de liderazgo de los servidores públicos mexicanos y su correspondencia con los indicadores de desempeño, por lo que relaciona directamente el resultado de los seguidores con la eficacia que ellos perciben del líder. Según este autor, debemos enfocarnos en el tipo de relación que se establece entre el directivo (líder) y los empleados que dependen directamente de él

Ayoub (2010) afirma que el liderazgo en la realidad no puede ser distante puesto que se encuentra presente en todos los niveles jerárquicos, en donde existe cercanía entre el líder y los seguidores, así que no se trata de la imagen que éste proyecta sino de una relación cercana.

Debido a ello, este autor hace una crítica al enfoque de la ciencia política. Afirma que, cuando la ciencia política habla de liderazgo, se identifica con un tipo de liderazgo distante e indirecto entre los seguidores, y esto es así -nos dice- porque desde la perspectiva de la ciencia política esta distancia es necesaria para el surgimiento del líder carismático, toda vez que la distancia no permite a los seguidores observar al líder en su verdadera dimensión humana, con sus defectos y virtudes sino a través de la imagen que proyecta, de manera que, surge el líder carismático.

Sin embargo, ante esos valiosos aportes, es necesario tener presente que si bien el estilo de liderazgo es importante para lograr resultados y motivar a los subalternos a aumentar los indicadores de desempeño, el concepto de gerencia va más allá de la mera conducción o de la conducción per se. En el caso de los gerentes públicos, se trata más bien del tipo de resultados que se logra en favor del bien común; es decir, no se trata de los objetivos del líder o gerente sino los de la organización, esto último conlleva a estudiar no sólo la relación entre el líder y los subalternos sino, además, los procesos y métodos para lograr que la organización alcance los objetivos y metas propuestos. En otras palabras, cuando se habla sólo de estilo de liderazgo, se corre el riesgo de dejar por fuera del análisis el resultado de la organización y de lo que trata ésta, precisamente, de usar el liderazgo para motivar a los subordinados hacia los objetivos de la organización. Por esta razón, el concepto de gerencia debe ser más amplio y utilizar además del liderazgo, el enfoque del proceso administrativo, para organizar y dirigir con eficiencia los recursos en el logro de esos objetivos eficaces.

De esta manera, se comparte lo dicho por Prats: la Administración Pública no es una hijuela de la ciencia política sino un producto de las escuelas de administración (Prats, 2005, p.82 citado por Cajas, año,p.10). Por lo tanto, aunque las ciencias políticas, la sociología, la psicología, entre otras, forman parte importante del estudio de la administración, la gerencia pública va más allá y une todo ello para cumplir las funciones que le son propias.

\section{Perfil administrativo del gerente público}

Tal y como lo expresan Robbins y Coulter (2011), en su libro "Administración", el gerente es la persona que coordina y supervisa el trabajo de otros para que se logren los objetivos de la organización. Agregan estos autores que el trabajo de un gerente no tiene que ver con los logros personales, sino con ayudar a otros a realizar su trabajo, y el trabajo que realizan todos es el que permite que la organización alcance las metas propuestas.

Llevado este planteamiento al sector público, se tiene que el plan de gobierno propuesto por el equipo o partido gobernante, debería darle al líder una visión de futuro clara para que éste, in- 
dependientemente de sus intereses personales, logre conjuntamente con sus subalternos, determinar una vía para alcanzar los objetivos ahí propuestos. Por supuesto que, como se ha dicho anteriormente, nada está sujeto a dogmas, de manera que si el líder o los subalternos consideran inviable dicho plan, el sistema democrático debe tener previamente establecidos los mecanismos para corregir aquello que en la práctica no conviene a los intereses de la colectividad. Pero en general, al gerente le corresponde liderar la definición de los derroteros, por donde debe llevar a la organización, por eso es necesario conocer cómo lograrlo, cuáles son las herramientas básicas de la administración que debe aplicar para conducir la organización (al logro de los objetivos en el contexto que dirige y sobre el entorno político que lo rodea).

Se arriba aquí, finalmente, al hecho de que para lograr la coordinación, la conducción y la ejecución de las demandas de la ciudadanía, las funciones del gerente público son necesariamente las mismas del gerente privado, las cuales, pueden y deben ser adaptadas en cada contexto.

Las funciones del gerente fueron propuestas por Henry Fayol a principios del siglo veinte y se refieren a la planeación, la organización, la dirección y el control (en Robbins et al., 2011). Aunque fueron pensadas para el sector privado, véase la descripción de ellas aplicadas al gerente del sector público.

Planear: el gerente define los objetivos y establece las estrategias para lograrlos, desarrolla los planes para integrar y coordinar las actividades. Es aquí donde se logra lo que el ex presidente Figueres llama gobernar, o encauzar el esfuerzo nacional hacia la formación de una patria mejor. Es en este punto donde el gerente debe tener claro el bien común, cuáles son los objetivos de la organización, los compromisos del partido y, específicamente, del gobierno de turno en favor de ese bien común.
Organizar: los gerentes deben determinar las tareas a realizar, quién las hace y cómo se llevarán a cabo; es decir, deben estructurar el trabajo para cumplir con lo planeado.

Tal y como lo veía el ex presidente Figueres, para su contexto, y que con un poco más de complejidad es la situación actual: organizar es "repartir el trabajo organizadamente, ver que cada funcionario cumpla con su deber, percibir las entradas de dinero y hacer los gastos llevando buenas cuentas (Figueres, 1956).

Dirección: en la función de dirección, el gerente trabaja con las personas motivando, resolviendo conflictos, influyendo, seleccionando las formas de comunicación más adecuadas, entre otras. Todo con el fin de cumplir con los objetivos propuestos.

Aquí sí se aplican todas esas teorías sobre liderazgo de las cuales nos habla Ayoub (2010): la sociología, la psicología y las ciencias políticas.

Control: finalmente, el control es la evaluación de todo lo actuado con el fin de asegurarse de que las cosas marchen según lo planeado. En el control se aplica tanto la evaluación a largo plazo, como lo que aquí se propone, el control continuo al estilo de Lewin (1946) con su método de investigación acción.

Aunado a lo anterior, el conocido investigador Henry Mintzberg (en Robbins et al., 2011) estudió comportamientos de gerentes y determinó que existen roles que los gerentes deben cumplir, como podrá observarse se aplican tanto al sector público como al privado:

Roles interpersonales: son aquellos que involucran personas (subordinados y personas ajenas a la organización) y que son de naturaleza simbólica: representante, líder, enlace.

Roles informativos: involucra reunir, recibir y transmitir información: monitor, difusor y portavoz 
Roles decisorios: conlleva la toma de decisiones o elecciones y son emprendedor, manejador de problemas, asignador de recursos y negociador.

Más recientemente, la empresa Dell Inc. (Robbins el al., 2011) realizó un estudio en donde determinó que para cumplir con sus funciones, el gerente requería contar con una serie de habilidades:

- Habilidades técnicas: son el conocimiento específico del trabajo y las técnicas para realizar competentemente las tareas.

- Habilidades Humanas: involucran la capacidad de trabajar bien con otras personas, tanto de manera individual como en grupo.

- Habilidades conceptuales: son aquellas que los gerentes utilizan para pensar y conceptualizar situaciones abstractas y complejas, donde pueden ver la organización como un todo, comprender la relación entre las diversas subunidades y visualizar cómo encaja su organización en su entorno general.

Si bien todas las habilidades son importantes para todos los gerentes, en este estudio, la empresa Dell Inc. hace una clasificación de esas habilidades, según la importancia que ellas tienen, para desempeñarse dentro de los diferentes niveles de administración en que esté ubicado el gerente - nivel alto medio o bajo-. Nos dice que el gerente de nivel alto, que es el de interés del presente trabajo, debe contar principalmente con habilidades conceptuales.

Efectivamente si revisamos las funciones del gerente descritas anteriormente, corresponde a los de alto nivel, involucrarse primordialmente en el plan estratégico, en el desarrollo de la visión y/o misión de la empresa y en la coordinación de todas las acciones que se desarrollan en la organización para lograr esos planes.
En síntesis, un gerente público requiere una serie de conocimientos administrativos mínimos para poder asegurarse algún grado de éxito en su función. Estos conocimientos se han dividido en dos grandes áreas que se muestran en la figura 2.

Figura2

\section{PERFIL ADMINISTRATIVO DEL GERENTE PÚBLICO}

Diferencias
entre lo público
y lo privado

Eficiencia y Eficacia
Fines sociales vs
lucro
Integralidad vs
dispersión

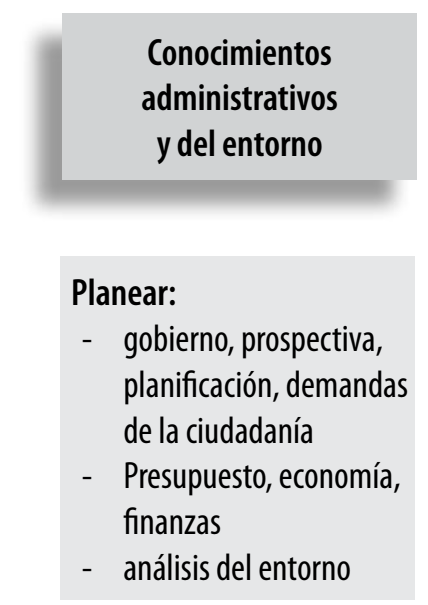

$$
\begin{aligned}
& \text { Organizar: } \\
& \text { procedimientos, división } \\
& \text { del trabajo, centralización, } \\
& \text { descentralización, grupos, } \\
& \text { equipos }
\end{aligned}
$$

\section{Dirigir: \\ liderazgo, análisis del \\ contexto político, toma \\ de decisiones}

\section{Controlar: \\ Controlar-Ejecutar- \\ Controlar-Evaluar}

Fuente: Elaboración Propia.

Como se desprende de la figura 2, no es conveniente, únicamente, estudiar el estilo de liderazgo con que se conduce a la organización, por- 
que se deja de lado la eficiencia y eficacia con que deben ser ejecutadas las acciones.

\section{CONCLUSIONES}

Se hace necesario contar con gerentes públicos capacitados en herramientas administrativas que les permitan liderar todo este proceso, además de la capacitación necesaria, para contar con una visión de la realidad nacional y de las demandas de los ciudadanos.

Más aún, no se debe olvidar que también se requiere de una verdadera estrategia nacional, para aprovechar las ventajas de la globalización y superar los obstáculos de visiones desintegradas, que podrían poner en desventaja la capacidad de negociación y de articulación del desarrollo de un país.

De lo contrario, se podría obtener un círculo perverso de países que tienen gerentes públicos que no entienden las verdaderas dimensiones de la gestión administrativa y, por lo tanto, no conocen cómo desarrollar una visión de futuro, lo cual a su vez resulta en países que no pueden dar seguimiento a sus grandes proyectos nacionales.

Estas carencias pueden dar lugar a situaciones en donde se justifica la no ejecución de nuevos programas, por la carencia de recursos, y la ejecución se convierte, en el mejor de los casos, en el mero mantenimiento de las actividades o procedimientos de rutina. Además, en estas circunstancias es muy probable que cuando se decide emprender nuevos proyectos, éstos van, generalmente, dirigidos a poblaciones muy pequeñas; es decir, son pequeños proyectos cuyas metas no tienen las dimensiones que la sociedad reclama.

Es, por esta razón, que en algunos países, los gerentes públicos de nivel medio y superior, utilizan varios años de su vida en conseguir educación administrativa formal. Uno de los más exigentes cursos de entrenamiento se realiza en Francia, donde los estudiantes de la Escuela Nacional de Administración (E.N.A), utilizan tres años de entrenamiento intensivo en cursos de gestión. Es necesario recordar que estos profesionales, antes de entrar a la E.N.A., ya tienen un título profesional avanzado, en áreas como derecho, medicina, pedagogía o ingeniería. En los países anglosajones, la profesión de gobierno, también se enseña a nivel de posgrado, esto significa que los estudiantes de gerencia pública son servidores públicos relativamente jóvenes, que han sido seleccionados para ocupar cargos superiores de administración (Duque, 2005).

Finalmente, y es de suma importancia reconocer que, no puede asumirse que el perfil de gerente público debe ser igual para todos los países. En países geográficamente muy grandes, las altas jerarquías del ejecutivo son puestos que probablemente requieren un profesional muy orientado a lo estratégico y menos a lo ejecutivo; dado que el gobierno debe tener muchos niveles: central, regional y local, por lo que este tipo de gerente se convierte en el gran coordinador, el visionario, el que marca el norte, el que aglutina el consenso o los acuerdos. En el caso de países pequeños, estos puestos se convierten en los gerentes públicos, que si bien son los visionarios, están también ligados a la coordinación de todo lo que se ejecute y por lo tanto, lo organizativo pasa a ser parte importante de sus funciones. Lo organizativo es tan cercano a su función, que si se deja de lado o si no se le da seguimiento, es probable que su eficacia se vea disminuida.

El desarrollo requiere de instituciones sólidas y la modernización debe pasar instituciones ágiles, capaces de ofrecer a la sociedad las demandas que solicita, lo cual sólo es posible con una buena gestión. El cambio se induce por la vía de quienes integran la organización, pero específcamente por la vía de las cúpulas; para lo cual éstas deben estar preparadas; es decir, contar con las herramientas administrativas para hacerlo. 
A partir de este pensamiento, se puede derivar que, si bien para obtener buenos resultados de una gerencia, tanto en el sector público como en el privado, es necesario contar con una serie de condiciones en el entorno, los buenos resultados no pueden darse, aún cuando el medio sea favorable, si el gerente desconoce cómo realizar la conducción del proceso.

El gerente público mal preparado implica un retraso al desarrollo, por el contrario, su mejora en el desempeño generaría, no sólo un mayor desarrollo de los programas a nivel nacional, sino también una mejora en la imagen del sector público y hasta en la del sistema político y en la gobernabilidad.

\section{REFERENCIAS}

Agüero, M. (2011, 16 de setiembre). Gerente financiero se va del ICE y deja sanción en suspenso. La Nación, p. 16A

Ayoub Pérez José Luis (2010). Estilos de Liderazgo y su eficacia en la administración pública mexicana. Lulu Enterprises: North Carolina,.

Bolaños, Rolando ( 2008) La Nueva Gerencia Pública, pág 144. Ciencias Económicas 26-No. 1: 2008 / 141-167 / ISSN: 0252-9521. Recuperado de: http://www.latindex.ucr.ac.cr/econ-2008-1/06-BOLANOS.pdf

Cabrero Mendoza, Enrique (2004). Del administrador al gerente público. Instituto Nacional de Administración Pública. INAP México. Recuperado de www.joseacontreras.net/

Cajas López Marco Tulio (2006, 10 de noviembre). ¿Por qué gerencia pública?: mitos y realidades. Recuperado de http://www.sercivil.go.cr/dgsc/documentos/cecades/gerencia-publica-mitos-y-realidades.pdf

Asamblea Legislativa (1949). Constitución Política de la República de Costa Rica. Editorial Costa Rica

COREC (1990). La Reforma del Estado en Costa Rica.

Daft, Richard (2011). Teoría y Diseño organizacional. CENGAGE Learning, Décima Edición.

Duque Fernando ( 2005, Mayo). El gerente público: un profesional para el desarrollo. Recuperado de: http://fernan- doduquecipol.blogspot.com/2009/03/el-gerentepublico-un-profesional.html

Figueres Ferrer José (1956). Cartas a un Ciudadano. Imprenta Nacional.

Lewin Kurt (1946). Groups, experiential learning and action research. Recuperado de: the encyclopedia of informal education (INFED)[www.infed.org]

Meoño Segura, Johnny (2010). Liderazgo administrativo para el cambio: Lo público y lo privado. Revista de Ciencias Económicas 28, Universidad de Costa Rica. Recuperado de: http://www.latindex.ucr.ac.cr/econ-282010/economicas_28_2_02.pdf

Meoño Segura, Johnny (2012). Análisis de los Procesos de Dirección, Planificación Gubernativa, Políticas Públicas y Reforma del Estado en Costa Rica. Revista de Ciencias Económicas, Universidad de Costa Rica. Recuperado de http://www.latindex.ucr.ac.cr/econ-30-1/economicas-30-1-01.pdf

Michael Barzelay (2000). The new public management: a bibliographical essay for Latin American (and other) scholars. London School of Economics and Political Science International Public Management Journal 3 (2000) 229-265. Recuperado de: http:// www.google.co.cr/url?sa $=t \& r c t=j \& q=\& e s r c=s \&$ frm $=1 \&$ source $=$ web $\& c d=1 \&$ ved $=0$ CFAQFjAA\& url=http $\% 3$ A\%2F\%2Fwww.ipmn.net\%2Findex. php\%2Fcomponent\%2Fdocman\%2Fdoc_ download\%2F42-the-new-public-management-a-bibliographical-essay-for-latina merican-and-other-scholars\&ei=DoLrT_ KHAoqk8QS2rJSmBQ\&usg=AFQjCNHW_nBILVXdWxh Al7S37bFlyTXaFA\&sig2=7SNbP0zWo3pURrlylzigig

MIDEPLAN (2012). Programa de Modernización del Estado. Recuperado de http://www.mideplan.go.cr/index php/la-organizacion-y-modernizacion/90/344?lang=

MIDEPLAN (2011, diciembre). Informe Noviembre 2010Noviembre 2011 Comisión de Eficiencia Administrativa (CEA). Area de Modernización del Estado. Recuperado de http://documentos.mideplan.go.cr/alfresco/d/d/ workspace/SpacesStore/b17b3c10-101c-44eeaf2c-8e2b82a08b23/Informe\%20CEA\%202010-\%20 2011\%20.pdf

Robbins Stephen y Coulter Mary (2011). Administración. Pearson. Tercera Edición.

Recibido: 29 de junio de 2012 Aceptado: 3 agosto de 2012 